\title{
Conductivity and charge depletion aging of resistive electrodes for high rate RPCs
}

\author{
Miguel Morales $^{\mathrm{a},{ }^{*}}$, Carlos Pecharromán ${ }^{\mathrm{b}}$, G. Mata-Osoro ${ }^{\mathrm{c}}$, L.A Díaz ${ }^{\mathrm{d}}$ and J.A. \\ Garzón $^{\mathrm{a}}$ \\ ${ }^{a}$ LabCAF, University of Santiago de Compostela, \\ Santiago de Compostela, Spain \\ ${ }^{b}$ Institute of Materials Science Research of Madrid (ICMM /CSIC), \\ Cantoblanco, Spain \\ ${ }^{c}$ École Nationale Supérieure de Chimie de Paris (ENSCP), \\ Paris, France \\ ${ }^{d}$ Nanomaterials and Nanotechnology Research Center (CINN/CSIC), \\ Oviedo, Spain \\ E-mail: miguel.morales@usc.es
}

ABSTRACT: Development of new electrodes is the key element for the improvement of the high rate Resistive Plate Chambers (RPC). In the particular case of resistive electrodes, the fabrication of these devices is a challenging problem from a material science point of view. The combination of resistivity, permittivity and stability requirements is really hard to satisfy for any known material. Respecting this, several materials have been found to be suitable for fabrication of resistive plates. In this work, we have carried out electrical characterizations of some of these materials that are currently used in RPCs or are solid candidates to be used as resistive plates for high rate RPCs. As a result, we have found strong evidences that ion conduction processes under moderate to high electric fields are able to drift high amounts of charge which are crucial to understand the degradation nature of the involved processes.

KEYWORDS: Resistive-plate chambers; Materials for gaseous detectors; Charge transport and multiplication in solid media; Charge induction.

\footnotetext{
* Corresponding author.
} 


\section{Contents}

1. Introduction $\quad 1$

2. Experimental setup $\quad 2$

3. Results and discussion $\quad 4$

3.1 Resistive electrodes permittivity $\quad \mathbf{4}$

3.2 Conductivity mechanism $\quad 5$

$\begin{array}{lr}3.3 \text { Ionic aging model } & 8\end{array}$

4. Conclusions $r$

\section{Introduction}

RPCs belong to the family of Parallel Plate ionizing gas detectors. Other well-known members of the family are the PPCs (Parallel Plate Chambers), with two metallic electrodes, and PMCs (Parallel Mesh Chambers), with multiple wire-mesh electrodes. The main difference and the key evolution issue over their relatives is that electrodes are not metallic materials but resistive plates. These specific electrodes allow the detector to work in sparkles operation mode. In this mode, discharge is quenched at an earlier stage, so the affected area is reduced. Furthermore, a smaller amount of charge per event also protects the electronics readout at the cost of amplifiers with greater gain. Besides dealing with smaller signals, another drawback of this RPCs operation mode is its dramatic counting rate limitation. For instance, the minimum ionizing particles (MIPs) rate capability of float glass RPCs at room temperature is limited to 2 $\mathrm{kHz} / \mathrm{cm}^{2}[1]$.

In nuclear and particle physics or astrophysics experiments RPCs have been widely used because of their versatility. Furthermore, their use in medical devices, as in Positron Emission Tomography (PETs), has also been foreseen in the near future [2]. Eventually, rate capability issues requiring the warming up of the glass electrodes [3] to achieve the needed rate capability are also feasible.

RPCs rate capability is mainly limited by the plate resistivity, which determines the efficiency decrease due to the electric field falling over the gas gap. In this regard, the most straightforward procedure for improving the rate capability or to speed up the charge drift is to employ relative low resistive electrodes. According to the so-called "DC model" for RPCs [4], rate capability is inversely proportional to the product of the plate resistivity times its thickness. Therefore, in order to improve the rate capability keeping the same plate thickness, conductivity needs to be notably increased. Taking into account the present values of low rate RPC plates resistivity, $\left(\rho \sim 10^{9}-10^{10} \Omega \bullet \mathrm{m}\right)$, materials with resistivity at least two orders of magnitude 
smaller are required. Unfortunately, it can be said that there is practically no natural material with such values of conductivity. In fact, ordinary materials can be classified as conductors $(\rho<$ $\left.10^{-2} \Omega \cdot \mathrm{m}\right)$ or insulators $\left(\rho>10^{9}-10^{10} \Omega \cdot \mathrm{m}\right)$. Materials with intermediate values of conductivity are really scarce. It is easy to be misled by the fact that conductor/insulator mixtures in different ratios or properly speaking, composites, present a wide range of resistivity values, 10 orders of magnitude, gradually distributed. However, real composites present percolation features, which make the transition incredibly sharp at a certain critical concentration, known as percolation threshold [5-6]. The transition width is so thin, that, in practice, is really hard to obtain materials with intermediate resistivity values. Materials with the appropriated values of resistivity are known as static-dissipative or lossy materials, are quite uncommon and most of the times they have to be specially designed to fulfil the requirements.

In addition to the above mentioned electric properties, high rate RPCs resistive plates should present long term stability. This requirement is crucial when dealing with RPCs assembled in large detectors. These devices hold a massive charge drift during long time periods. Furthermore, besides resistivity, other properties, as permittivity, dielectric strength (breakdown field) or current stability have to be considered. For instance, the charge that will pass through the RPC TOF at the CBM experiment [7] at FAIR, along the expected 5 years lifetime is around $1 \mathrm{C} / \mathrm{cm}^{2}$. This value, which is usually taken as a benchmark reference for aging tests, is definitely a large amount of charge, and can easily damage the plate material.

\section{Experimental setup}

The materials considered as resistive plates appear in table 1. Some of them have already been used in RPCs, both for low and high rate applications. Besides, two new materials designed for high rate have been added. The first one is a ceramic/metal composite (labelled as Mullite/Mo) and the second is a ceramic/oxide composite. Details about preparation of both types of composites can be found in [8]. Results of the analysis about resistivity and charge depletion properties are presented for several resistive plates materials. Moreover, a model has been developed showing the ionic conductivity component and the current dependence with electric field and time.

Table 1: Set of tested materials. Glass and polymers are selected from different RPC experiments, while ceramic materials are made in our laboratory.

\begin{tabular}{lll} 
Material Type & \multicolumn{1}{c}{ Material } & \multicolumn{1}{c}{ Provider } \\
\hline Glass & Soda Lime Silicate Glass (SLS Glass) & $\begin{array}{l}\text { Hades experiment, } \\
\text { GSI(Darmstadt) }\end{array}$ \\
& Low Resistive Silicate Glass (LR S Glass) & Tsinghua University \\
Polymer & Bakelite (High Pressure Laminate) & CMS experiment \\
Ceramics & Mullite/Mollybdenum (Mullite/Mo) & ICMM/CSIC, Madrid \\
& Ferrite Ceramic & ICMM/CSIC, Madrid
\end{tabular}




\section{Computer}

\section{Temperature}

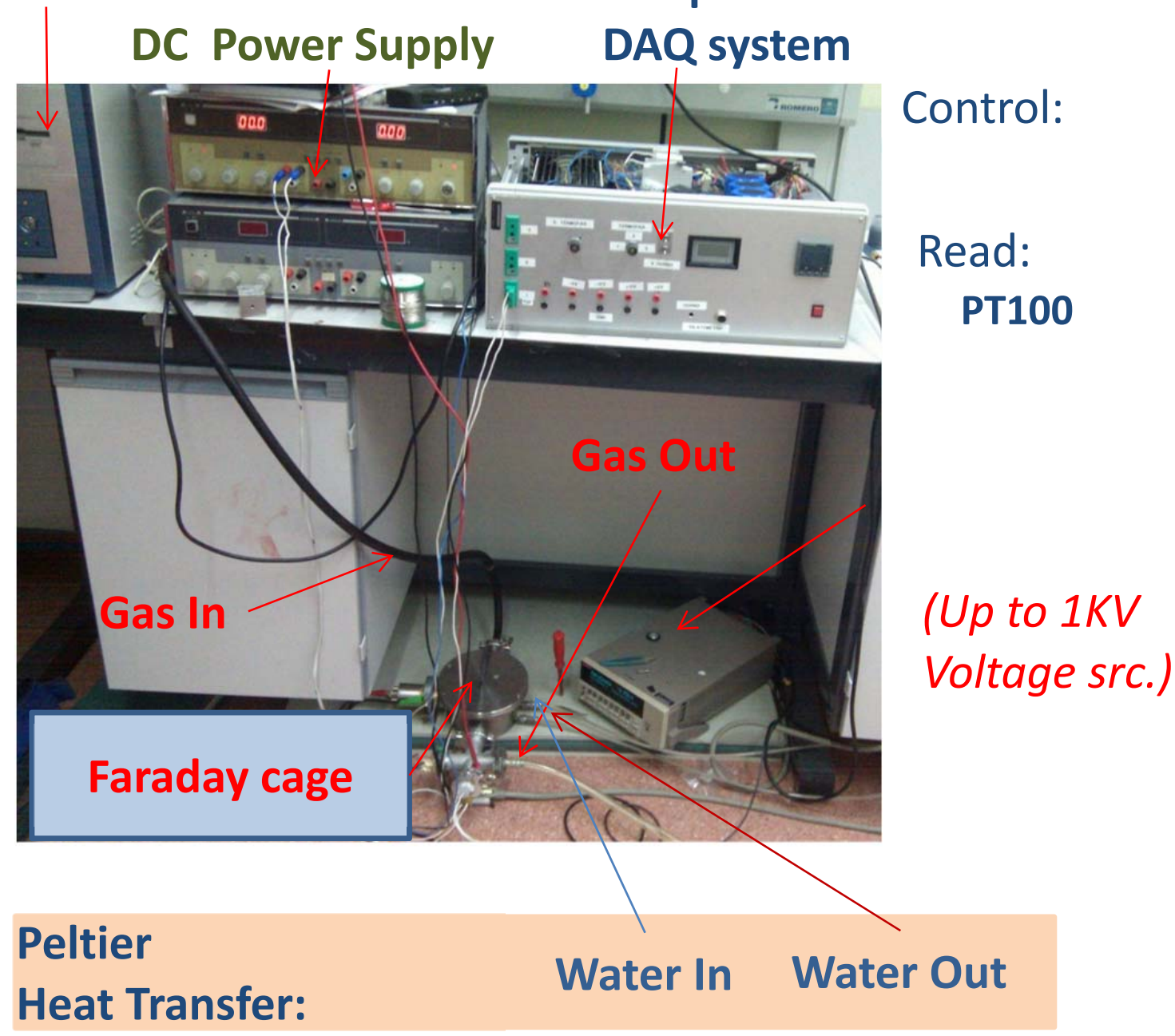

Figure 1. Picture of the experimental setup for electric characterization.

Detectors were tested in controlled temperature and gas environments. The experimental setup can be seen in figure 1. In order to check the RPC's stability under an applied electric field, measurements of the relevant magnitudes were taken for extended time periods. Also, to achieve a meaningful amount of charge transferred keeping the experiments within a reasonable time span, the materials were conveniently polished to a very thin thickness $(<100 \mu \mathrm{m})$ and the temperature was increased when needed. All the measurements were taken inside a Faraday cage to screen external electromagnetic interferences. Gas environment was under control, keeping $\mathrm{N}_{2}$ steadily flowing inside the box to avoid external charge carriers additions, as $\mathrm{O}_{2}$ or humidity. Temperature values were acquired regularly by a PT100 thermoresistance in near contact to the sample and regulated by a Peltier cell controlled by computer. Different magnitudes of electric field were applied to the resistive plates by the electrometer itself (Keithley 6517A) using painted silver as electrodes. Permittivity values were measured by an Agilent LCR 4284A impedance analyzer instead of the electrometer. 


\section{Results and discussion}

In this work, different electric measurements have been carried out on five different materials which currently are or could be used as ressistive plates. In most of the experiments we have kept constant the electric field vs time, neglecting transitorial relaxation. This regime can be considered as DC (Direct Current) from the conductivity point of view (let's note that DC conductivity regime is a different concept as the "DC-model" presented in reference [4]). Permittivity measurements have been the only exception. Actually, AC (Alternate Current) complex impedance measurements were performed on all the samples from 20 to $1 \mathrm{MHz}$.

\subsection{Resistive Electrodes Permittivity}

Besides resistivity, permittivity plays a remarkable role in the electromagnetic field distribution between resistive plates and gas gaps. Actually, the conduction processes associated to ionization phenomena develop in a broad time. In this AC regime, the impedance is given by its complex expression where the capacitive term is preponderant at higher frequencies. Therefore, dielectric discontinuities in the detector could substantially modify the field distributions. In this sense, dielectric constant values of classic RPC plate materials, Bakelite and SLS Glass, are very low and similar, so the permittivity value has usually been ignored. But when dealing with high rate RPCs, it is necessary to keep in mind that permittivity of the resistive plates has a strong influence at three remarkable points corresponding to the detector behavior:

1) Gas gap electric field recovery time. This magnitude is linked with the relaxation time, $\tau=R \bullet C$ (Resistance times Capacity), i.e. the required time to dissipate the capacitor stored charge.

2) Resistive electrode stored energy. It is clear that lower values are safer for the electronics front end, as well as the material. Because the energy is proportional to the resistive electrode capacity, permittivity should be kept as small as possible.

3) Oppositely to the former point, continuity of the electric field displacement at the plate/gas interface induces an additional consideration. For a properly designed RPC, it is required that most of the voltage will drop into the gas gap. In this sense the condition of the electric displacement continuity states [9]: $D=\varepsilon_{\mathrm{g}} E_{g}=\varepsilon_{p} E_{p}$, where $\varepsilon$ is the permittivity and $E$ the electric field for the gas gap (g) and the resistive plate (p). Therefore, the bigger the inequality of $\varepsilon_{p}>\varepsilon_{g}$, the higher the voltage drop in the gap.

As a result of these three considerations it is not obvious to determine which the optimal permittivity plate values are. 


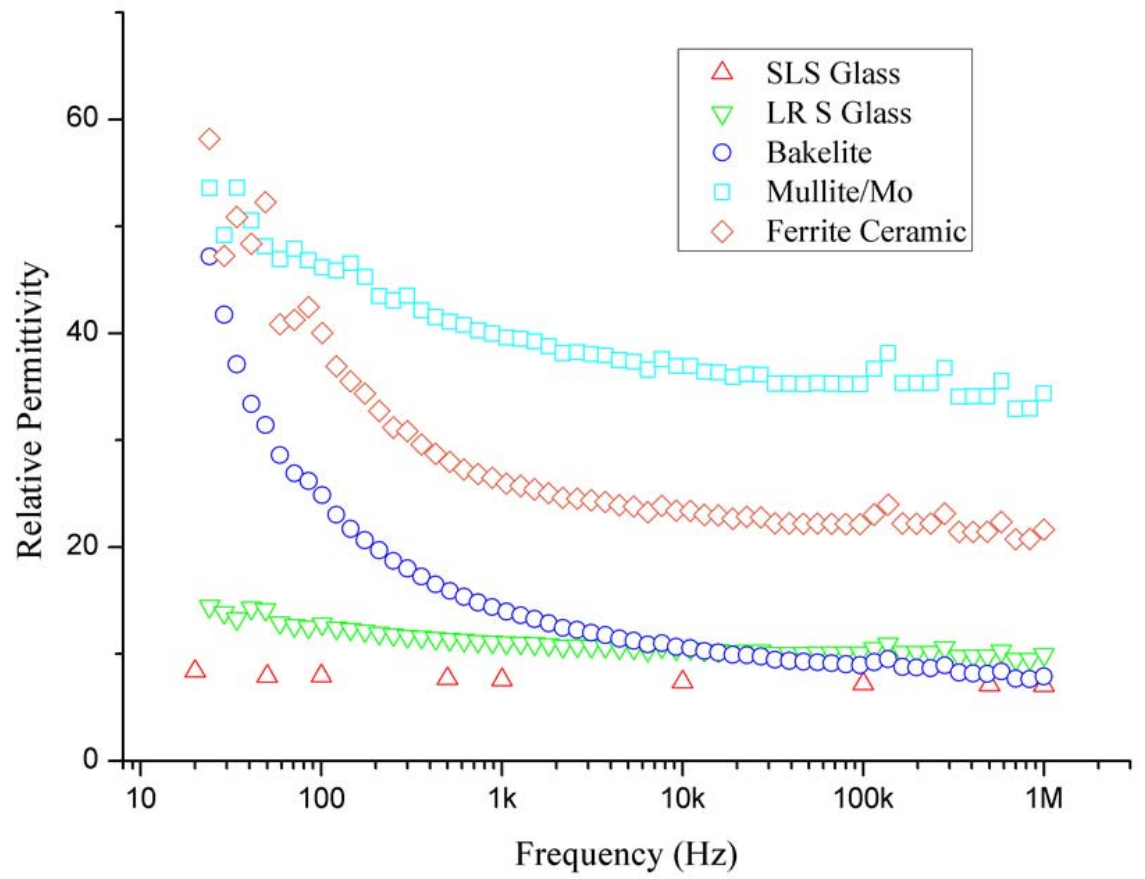

Figure 2. Permittivity of different high rate RPC plate materials measured in an LCR (Inductance Capacitance and Resistance) meter up to $1 \mathrm{MHz}$ using Ag paint circular electrodes.

In Figure 2 the permittivity vs frequency has been plotted for three materials employed as resistive electrodes (SLS Glass, LRS Glass and Bakelite) and two ceramic composites with conductivity values tailored to the high rate RPC specifications (Ferrite composites and $\mathrm{Mu} / \mathrm{Mo}$ cermet). For the first case, all the materials have relative permittivity values around $\varepsilon_{r} \sim 10$ at higher frequencies while new composites exhibit moderately larger values. For all the tested working RPC plates, SLS Glass, Bakelite and LR S Glass permittivity frequency dependence can be associated to relaxation processes, probably due to some blocking resistance. However it is not clear if this process is due to an electrode blocking, as it happens in ion conducting processes, or to a grain boundary resistance due to a thin insulating layer covering the conductive phase in a composite.

In any case, all dielectric constant are of the same order of magnitude, therefore, it is not expected that differences in permittivity of the selected samples will play a main role in the electric response of resistivity plates.

\subsection{Conductivity mechanism}

In order to determine the conductivity mechanism for each material, conductivity measurements vs. applied field and temperature were carried out in the previously described experimental setup. For sake of conciseness, only data corresponding to LR S Glass have been plotted in figure 3. 


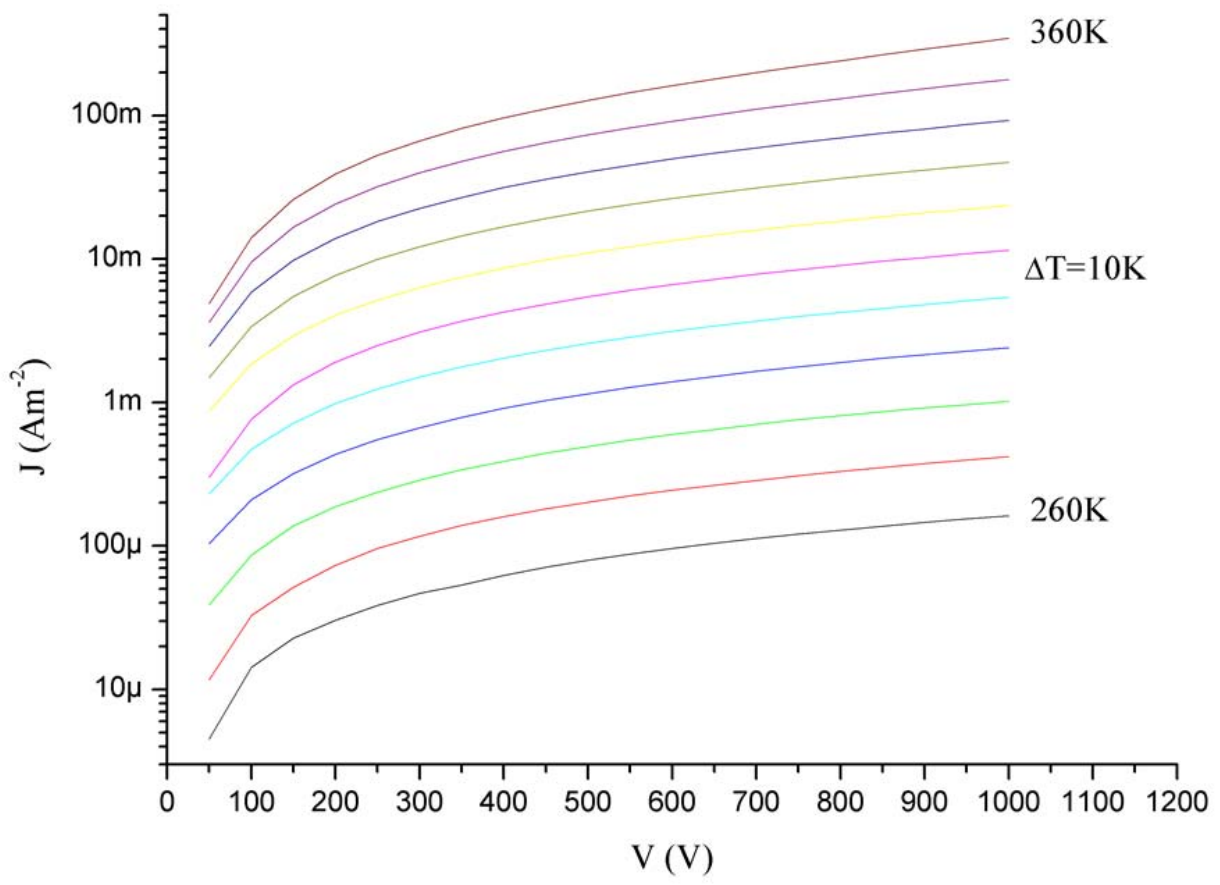

Figure 3. Conductivity of LR S Glass, $0.7 \mathrm{~mm}$ thick sample, on vacuum $\left(10^{-6} \mathrm{mbar}\right)$.

Several conduction models have been tried to fit on these data, but only ion conduction model under strong fields [10] or faradaic current seem to fit data of figure 3 . The current density definition given by:

$$
J=n q \mu
$$

where $n$ is the number of carriers per unit of volume, $q$ is the ion charge and $\mu$ is the mobility, or the velocity attained by these ions within an electric field. The average drift velocity of an ion affected by a high electric field is given by:

$$
\mu=2 l v_{0} e^{-\frac{W}{k T}} \sinh \left(\frac{q E l}{2 k T}\right)
$$

where $l$ is the so called jump length, $v_{0}$ is the oscillation frequency of the ion in the potential well $\left(\sim 10^{13} \mathrm{~s}^{-1}\right)$, W is the potential barrier and $E$ is the "microscopic" electric field seen by the ions. Finally, the current density, $J$, can be written as a function of the applied electric field as:

$$
J=2 l q v_{0} n e^{-\frac{W}{k T}} \sinh \left(\frac{q E l}{2 k T}\right)
$$

According to this law, the current density has a linear dependence on $E$ at low electric fields and follows an $E^{3}$ tendency with increasing values of $E$.

All the considered materials fit to this model but ferrite ceramic. Measured current densities $J$ as a function of the applied electric field, together with the results of the curve fitting are shown in figure 4 : 


$$
J=C_{1} \sinh \left(C_{2} E\right)
$$

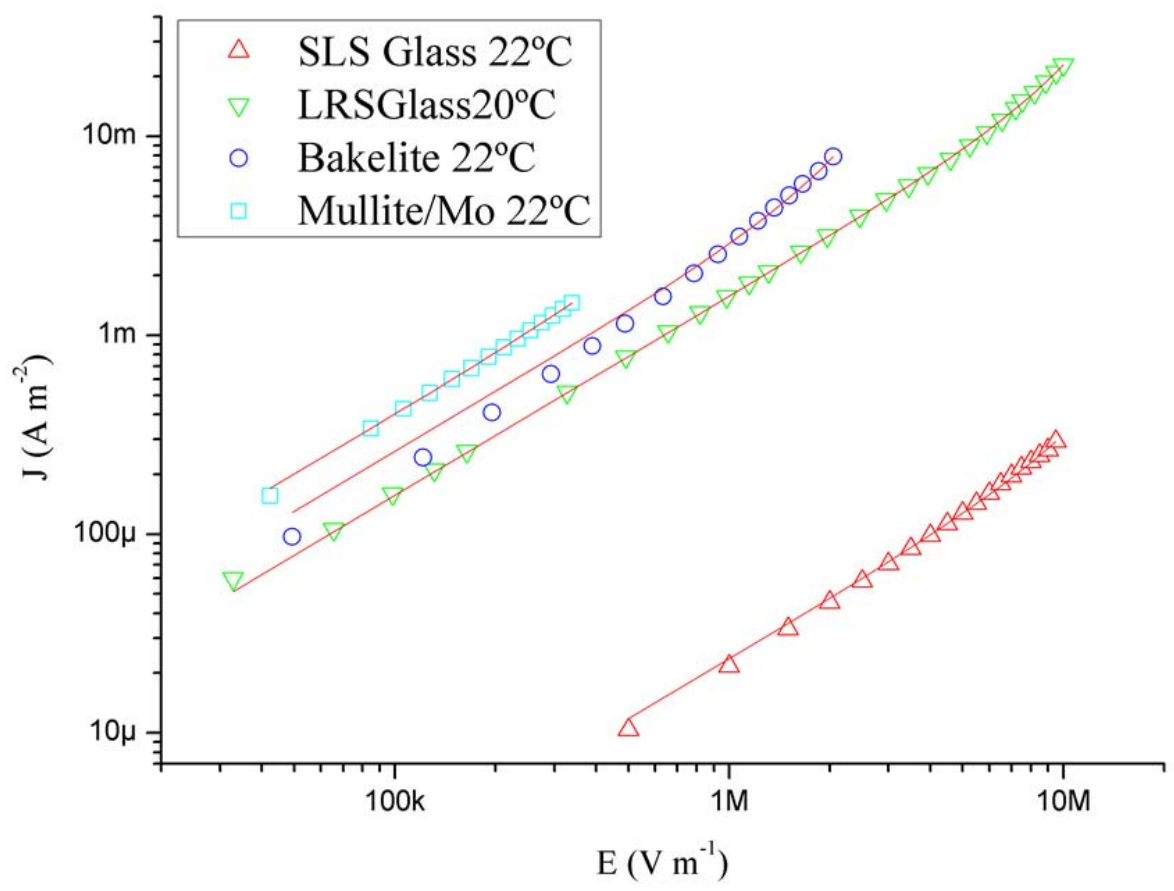

Figure 4. Current density as a function of the applied electrical field measured for some of the materials tested. The dashed lines show the results of the fitting done to the curve explained in the text.

According to Eq. (3) the fitting coefficients $C_{1}$ and $C_{2}$ are:

$$
C_{1}=2 l q v_{0} n e^{-\frac{W}{k T}} \quad C_{2}=\frac{q l}{2 k T}
$$

And consequently resistivity, $\rho$, and mean free path, $l$, are:

$$
\rho \propto \frac{1}{C_{1} C_{2}} \quad l=\frac{2 k T}{q} C_{2}
$$

Table 2 shows the results obtained for all the analysed materials assuming the ionic conductivity model. Only SLS glass presents resistivity values of the order of $10^{10} \Omega \mathrm{m}$. This is a material currently used for low rate RPC plates, and its resistivity is, at least, two orders of magnitude larger than those designed for high rate RPCs. It is also interesting to observe that the mean free length of the mullite/Mo plate is significantly higher $\left(10^{-7} \mathrm{~m}\right)$ than for the rest of materials. This is a well-known effect caused by the amplification of the electric field that appears in metal/insulator composites being close to the percolation threshold [11]. 
Table 2: Results of the fitting of the ionic current model referenced in the text to different materials; both resistivity $(\rho)$ and mean free length $(l)$ have been derived from the function coefficients, $C_{1}$ and $C_{2}$.

\begin{tabular}{lllll} 
& \multicolumn{1}{c}{$\rho(\Omega \mathrm{m})$} & \multicolumn{1}{c}{$C_{1}\left(\mathrm{~A} \mathrm{~m}^{-2}\right)$} & \multicolumn{1}{c}{$C_{2}\left(\mathrm{~m} \mathrm{~V}^{-1}\right)$} & $l(\mathrm{~m})$ \\
\hline SLS Glass & $(4.2 \pm 0.7) \cdot 10^{10}$ & $(1.7 \pm 0.2) \cdot 10^{-4}$ & $(1.4 \pm 0.1) \cdot 10^{-7}$ & $(7.0 \pm 0.6) \cdot 10^{-9}$ \\
LR S Glass & $(6.4 \pm 0.1) \cdot 10^{8}$ & $(1.00 \pm 0.01) \cdot 10^{-2}$ & $(1.57 \pm 0.01) \cdot 10^{-7}$ & $(7.9 \pm 0.2) \cdot 10^{-9}$ \\
Bakelite & $(3.8 \pm 0.8) \cdot 10^{8}$ & $(3.3 \pm 0.4) \cdot 10^{-3}$ & $(7.8 \pm 0.7) \cdot 10^{-7}$ & $(4.0 \pm 0.4) \cdot 10^{-9}$ \\
Mullite/Mo & $(2.5 \pm 0.3) \cdot 10^{8}$ & $(2.0 \pm 0.1) \cdot 10^{-3}$ & $(2.0 \pm 0.1) \cdot 10^{-6}$ & $(1.01 \pm 0.08) \cdot 10^{-7}$
\end{tabular}

\subsection{Ionic aging model}

Ion conduction in DC regime (faradaic current) could present blocking process when the employed electrodes hinder ion diffusion mechanisms. In the herewith considered glassy systems, one can assume that alkaline or alkaline earth cations can diffuse along the silicate framework under strong electric fields. However, once these carriers reach the copper, silver, gold or platinum electrode, electrochemical reactions take place and cations precipitate in the neighbourhood of the cathode. Therefore, one can expect charge depletion process in these materials for large time periods under strong electric fields. In this sense, we performed aging experiments for different materials and we have analysed the results assuming the following statements:

1) RPC plates could have small but measurable ion conductivity with blocking electrodes, so ions move to the corresponding electrode when a DC current is applied.

2) When a carrier reaches the electrode it is blocked there.

In any case, all carriers arriving at the interface do not further participate in the ion conduction process. Then, the carrier density loss is related to the faradaic current through equation:

$$
\frac{d n}{d t}=-\frac{J}{q d}
$$

Where $d$ is the sample thickness. In case of low to moderate electric field, $J$ can be approximated as Eq. (3).

$$
J(t)=l^{2}(t) n(t) \frac{E v_{0} q^{2}}{k T} e^{-\frac{W}{k T}}
$$

According to the results in Figure 5, the dependence of the mean free path with the time has been explicitly introduced, while the remaining terms are assumed to remain constant. As the variation of $l(t)$ is expected not to be very high ( $25 \%$ for 1 month, for SLS glass) the following second degree polynomial time dependence has been added:

$$
l(t)=l_{0}\left(1-\frac{t}{\tau_{1}}+\frac{t^{2}}{\tau_{2}^{2}}\right)
$$


This is a phenomenological approach in which it is assumed that the mean free path does not notably change inside the material at atomic scale even when part of the carriers are being removed from the structural framework.

Integrating $n(t)$ from Eq. (7):

$$
J(t) \approx J_{0}\left[1-2 \frac{t}{\tau_{1}}+t^{2}\left(\frac{1}{\tau_{1}^{2}}+\frac{2}{\tau_{2}^{2}}\right)\right] e^{-\frac{t}{\tau_{n}}\left[1-\frac{t}{\tau_{1}}+\frac{t^{2}}{3}\left(\frac{1}{\tau_{1}^{2}}+\frac{2}{\tau_{2}^{2}}\right)\right]}
$$

Where parameter $\tau_{n}$ is related to the carrier leakage rate and $\tau_{l}$ to the matrix deformation rate.

$$
\tau_{n}=\frac{d k T e^{\frac{W}{k T}}}{l_{0}^{2} v_{0} q E}
$$

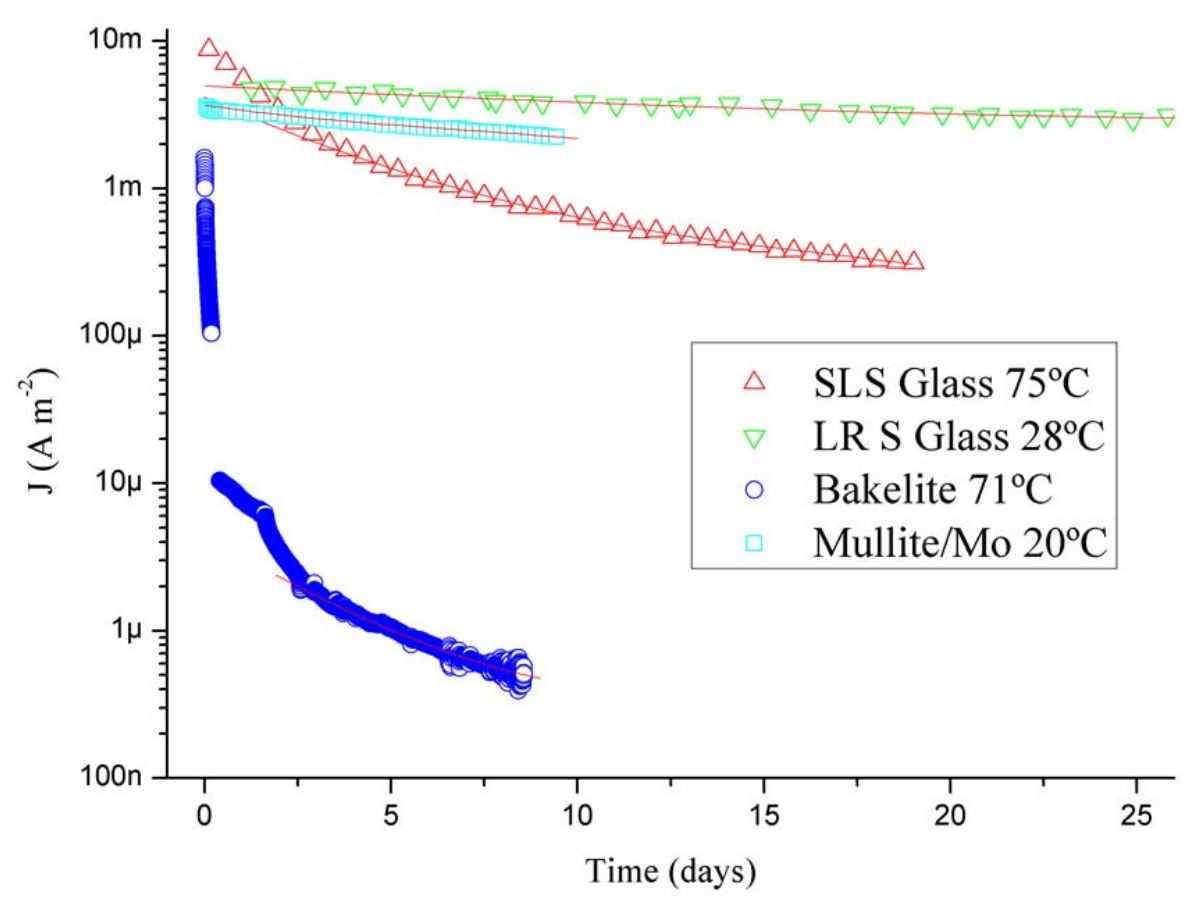

Figure 5. Density current vs. time behaviour of some of the analysed materials. The dashed lines show the results of the data fitting to the ionic conductivity model. As the measurements taken during the first days of the experiment are affected by polarizations and other transitory effects they have been excluded from the curve fitting.

Figure 5 shows the results of the fitting for several materials and table 3 shows the values of the fitted parameters; in general, there is a good agreement between data and model. Larger values of $\tau_{n}$ stand for longer times to lose conductivity due to the charge drift. For instance, SLS Glass looks to be a very poor candidate for long life high rate RPCs. On the other hand, LRS Glass has a poor agreement with the model (uncertainties are very high), since that the loss of conductivity is mainly not ionic. 
Table 3: Fitting parameter of the ionic conductivity aging model for several materials. Longer times and a worse fitting to the model stand for a poorer ionic conductor and a better high rate RPC candidate.

\begin{tabular}{lllll} 
& $\mathrm{J}_{0}\left(\mathrm{~A} \mathrm{~m}^{-2}\right)$ & $\tau_{l}$ (days) & $\tau_{2}$ (days) & $\tau_{n}$ (days) \\
\hline SLS Glass & $4.2 \pm 0.6 \cdot 10^{-3}$ & $26.3 \pm 4.2$ & $32 \pm 8$ & $5.6 \pm 0.9$ \\
LR S Glass & $4.9 \pm 0.3 \cdot 10^{-3}$ & $100 \pm 100$ & $91 \pm 25$ & $200 \pm 1200$ \\
Bakelite & $4.45 \pm 0.06 \cdot 10^{-6}$ & $14.7 \pm 0.6$ & $31.3 \pm 0.5$ & $48 \pm 2$ \\
Mullite/Mo & $3.7 \pm 0.2 \cdot 10^{-3}$ & $333 \pm 667$ & $20 \pm 4$ & $12 \pm 2$
\end{tabular}

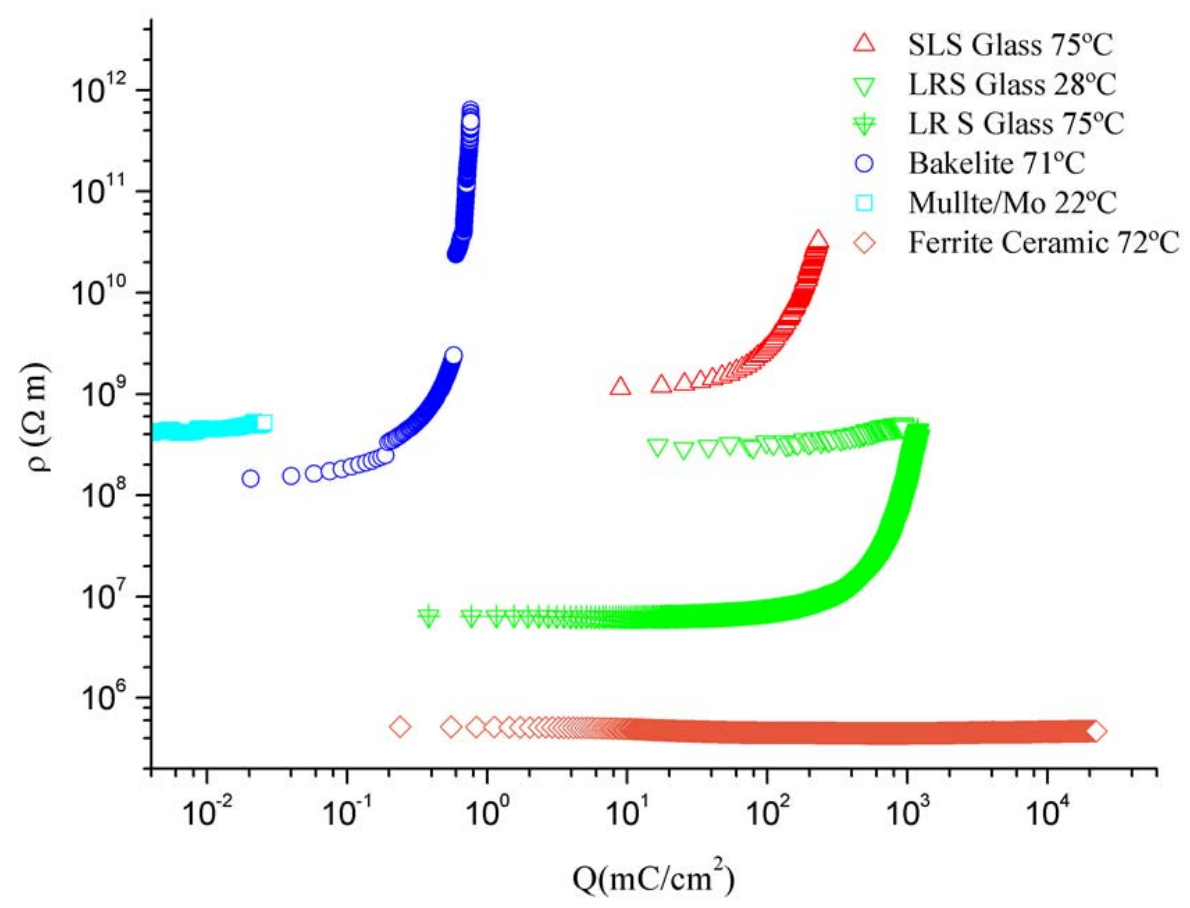

Figure 6. Resistivity vs. transferred charge measured for several materials and different temperatures.

It is well know that Bakelite is a water molecules $\mathrm{H}+$ ionic conductor [12]. Consequently, Bakelite in a water vapour free environment, presents charge depletion behaviour at $1 \mathrm{mC} / \mathrm{cm}^{2}$. Identically, SLS Glass run out of $\mathrm{Na}+[13]$ cations at $100 \mathrm{mC} / \mathrm{cm}^{2}$, while LR S Glass at $\mathrm{T}=75^{\circ} \mathrm{C}$ extinguishes its charge carrier ions a $1000 \mathrm{mC} / \mathrm{cm}^{2}$.

The case of the LR S Glass is not as simple as the ordinary SLS glass. It should be noted how for $\mathrm{T}=28^{\circ} \mathrm{C}$, charge depletion process has disappeared. On the other hand, LRS Glass presents a poor agreement with the $\mathrm{J}(\mathrm{E})$ model (figure 5). This case is very remarkable; we attribute this fact to a mixed ionic-electronic conduction mechanism for this material. In this sense, all the electronically transferred charge will not be depleted and consequently the 
proposed model will not fit so fine to this material. An important consequence is that all materials presenting a high amount of electron conductivity will exhibit a notable stability for long operating times.

As well as LR S Glass at $\mathrm{T}=28^{\circ} \mathrm{C}$, Mullite/Mo, and Ferrite/Ceramic doesn't show any charge depletion phenomena, probably related to an electronic conduction mechanism. However an electronic conduction process does not guarantee the desired resistivity stability for longer times under strong applied fields. Besides the conductivity degradation, atomic framework can be damaged too by the effect of cation migration [14][15], as it is the case of Mullite/Mo composites, which present sudden dielectric breakdown processes.

It is also remarkable to note that ferrite ceramic doesn't show any resistivity rise even after have delivered $22000 \mathrm{mC} / \mathrm{cm} 2$, so ferrite ceramic and LRS glass (for temperatures not higher than $\mathrm{T}=28^{\circ} \mathrm{C}$ ) are good candidates to be used as resistive electrode in high rate RPCs long run detectors.

\section{Conclusions}

A systematic electric characterization (AC complex impedance vs. frequency, DC conductivity vs. temperature, field and time) has revealed to be essential to determine the viability of any material for the fabrication of high rate RPC plates. Additionally, a detailed analysis of data acquired in the electrical measurement cell is able to identify the undergoing conduction mechanisms. In the case of low rate RPC materials (conventional glasses and Bakelite), charge is assumed to be carried by ions. Therefore, the response of electric current vs. the electric field for these materials was successfully fit to a conventional DC ion conduction mechanism. While these materials can work in a low rate RPC plate for very long time without any serious degradation, in the case of a high rate RPC, charge diffusion process may cause a depletion phenomenon, and therefore, a strong time dependant resistivity degradation of the material will appear. In this work we have experimentally proved the mentioned ionic aging. Because the ultimate cause of the charge depletion process is the presence of blocking electrodes, we have concluded that electron conductive materials do not suffer from resistivity aging. Therefore we propose to employ materials with a large contribution of DC electron conductivity for high rate RPC plates.

\section{References}

[1] V. V. Parkhomchuck, Yu. N. Pestov and N. V. Petrovykh, A spark counter with large area, NIM A. (93), 1971.

[2] M. Couceiro, et al., RPC-PET: Status and perspectives, NIM A, (580) 2, 2007.

[3] C. Gustavino, et al., Performance of glass RPC operated in avalanche mode, NIM A 527(3), 2004.

[4] G. Carboni, et al., A model for RPC detectors operating at high rate, NIM A (498)1-3, 2003.

[5] S. Kirkpatrick, Percolation and Conduction, Rev. Modern Phys. 1973, 45, 574.

[6] D. Stauffer, Scaling theory of percolation clusters, Physics Reports, Volume 54, Issue 1, July 1979, Pages 1-74. 
[7] I. Deppner, et al., The CBM time-of-flight wall, NIM A (661) Supplement 1, 2012.

[8] Moya, J.S. , Lopez-Esteban, S. , Pecharromán, C., The challenge of ceramic/metal microcomposites and nanocomposites, Progress in Materials Science, Volume 52, Issue 7, September 2007, Pages 1017-1090

[9] Ammosov, V., V. Korablev, and V. Zaets, Electric field and currents in resistive plate chambers, NIM A 401(2-3), 1997.

[10] Hyde, J.M. and M. Tomozawa, The Relationship Between The Dielectric-Relaxation And The HighField Conduction Of Glasses. Physics and Chemistry of Glasses 27(4), 1986.

[11] Pecharromán, C. , Esteban- Betegón, F., Jiménez, R, Electric field enhancement and conduction mechanisms in Ni/BaTiO 3 percolative composites, Ferroelectrics, Volume 400, Issue 1, 2010, Pages 81-88.

[12] Va'vra, J.; , Attempt to correlate the ionic model with observations in BaBar RPC chambers and $R \& D$ tests, Nuclear Science, IEEE Transactions on , vol.51, no.5, pp. 2145- 2150, Oct. 2004

[13] Natrup FV, et al., Cation diffusion and ionic conductivity in soda-lime silicate glasses. Phys Chem Chem Phys. 2005 Jun 7; 7(11):2279-86.

[14] E Thommerel, et al., Relations between microstructure, electrical percolation and corrosion in metal-insulator composites, Materials Science and Engineering: A, Volume 328, Issues 1-2, May 2002, Pages 67-79

[15] Mata-Osoro, G., et al., Faradaic current in different mullite materials: single crystal, ceramic and cermets. International Journal of Materials Research. 103(4): p. 408-411, 2012. 